\title{
The neurology of COVID-19
}

\section{La neurología de COVID-19}

\author{
Carlos Cuevas-García, ${ }^{1}$ Alejandra Calderón-Vallejo, ${ }^{1}$ Laura Berrón-Ruiz ${ }^{2}$
}

\begin{abstract}
The clinical manifestations of COVID-19 are reminiscent of those of acute respiratory distress syndrome induced by cytokine release syndrome and secondary hemophagocytic lymphohistiocytosis that is observed in patients with other coronaviruses such as SARS-CoV and MERS-CoV. Neurologists face the challenge of assessing patients with pre-existing neurological diseases who have contracted SARS-CoV-2, patients with COVID-19 who present neurological emergencies, and patients who are carriers of the virus and have developed secondary neurological complications, either during the course of the disease or after it. Some authors and recent literature reports suggest that the presence of neurological manifestations in patients who are carriers of SARS-CoV-2 may be associated with a greater severity of the disease.
\end{abstract}

Key words: COVID-19; SARS-CoV-2; Acute respiratory distress syndrome; Cytokine storm; Neurological disorders

Este artículo debe citarse como: Cuevas-García C, Calderón-Vallejo A, Berrón-Ruiz L. La neurología de COVID-19. Rev Alerg Mex. 2020;67(4):338-349

ORCID

Carlos Cuevas-García, 0000-0002-3314-5893; Alejandra Calderón-Vallejo, 0000-0002-6367-9124;

Laura Berrón-Ruiz, 0000-0002-3290-8705

${ }^{1}$ Instituto Mexicano del Seguro Social, Centro Médico Nacional Siglo XXI, Hospital de Especialidades, Ciudad de México, México ${ }^{2}$ Secretaría de Salud, Instituto Nacional de Pediatría, Unidad de Investigación en Inmunodeficiencias, Ciudad de México, México
Recibido: 2020-11-20

Aceptado: 2020-11-22

DOI: $10.29262 /$ ram.v67i4.828

Correspondencia: Carlos Cuevas-García.

cacu61152@hotmail.com 


\section{Resumen}

Las manifestaciones clínicas de COVID-19 recuerdan las del síndrome de insuficiencia respiratoria aguda inducido por el síndrome de liberación de citocinas y la linfohistiocitosis hemofagocitica observada en pacientes con otros coronavirus como SARS-CoV y MERS-CoV. Los neurólogos tienen el reto de evaluar pacientes con enfermedades neurológicas preexistentes que contraen SARS-CoV-2, pacientes con COVID-19 que presentan emergencias neurológicas y pacientes portadores del virus que desarrollan complicaciones neurológicas secundarias, durante el curso de la enfermedad o posterior a la misma. Algunos autores y reportes en la literatura recientes sugieren que las manifestaciones neurológicas en pacientes portadores de SARSCoV-2 pueden asociarse con mayor gravedad de la enfermedad.

Palabras clave: COVID-19; SARS-CoV-2; Síndrome de insuficiencia respiratoria aguda; Tormenta de citocinas; Afectaciones neurológicas

\author{
Abreviaturas y siglas \\ $\mathrm{CD}$, células dendríticas \\ $\mathrm{E}$, envelope \\ ECA2, enzima convertidora de angiotensina 2 \\ EVC, enfermedad vascular cerebral \\ INF, interferón \\ LHHS, linfohistiocitosis hemofagocitica secundaria \\ $\mathrm{M}$, membrana \\ MERS, síndrome respiratorio del medio oriente \\ $\mathrm{N}$, nucleocápside
}

\author{
$\mathrm{RM}$, resonancia magnética \\ S, spike \\ SARS, síndrome respiratorio agudo severo \\ SARS-CoV-2, severe acute respiratory syndrome co- \\ ronavirus 2 \\ SIRA, síndrome de insuficiencia respiratoria aguda \\ SLC, síndrome de liberación de citocinas \\ SNC, sistema nervioso central \\ EVC, enfermedad vascular cerebral
}

\section{Antecedentes}

La Organización Mundial de la Salud declaró la enfermedad respiratoria causada por el virus SARSCoV-2 (severe acute respiratory syndrome coronavirus 2) como pandemia, el 11 de marzo de $2020 .^{1}$

Hasta el día 2 de noviembre se habían reportado, 46838194 de casos confirmados y 1204003 de muertes en el mundo y México reportó 929392 casos confirmados y 91895 muertes para la misma fecha. $^{2}$

La naturaleza altamente transmisible del SARSCoV-2, su presencia en portadores asintomáticos y el amplio espectro de la enfermedad constituyen un enorme reto para todos los sistemas de salud en el mundo.

Los síntomas de la infección por SARS-CoV-2 se manifiestan después de un periodo de incubación de cinco días (dos a 14 días), los más comunes son fiebre, tos, fatiga, cefalea, hemoptisis y disnea; en casos graves, neumonía, alteraciones cardiacas y falla multiorgánica. Las comorbilidades asociadas, edad avanzada, enfermedades cardiovasculares o respiratorias preexistentes, obesidad y diabetes mellitus se han asociado con mayor gravedad y mal pronóstico. ${ }^{3}$

Las manifestaciones clínicas de COVID-19 (coronavirus disease 19) semejan las manifestaciones de SIRA (síndrome de insuficiencia respiratoria aguda) inducido por el síndrome de liberación de citocinas (SLC) y la linfohistiocitosis hemofagocitica secundaria (LHHS) que se han observado en pacientes con otros coronavirus como SARS-CoV y MERS-CoV (Middle East respiratory syndrome coronavirus) ${ }^{4}$

El síndrome respiratorio agudo es la característica clínica más importante de la enfermedad; SARs-CoV-2 puede afectar a otros órganos y sistemas, incluyendo al sistema nervioso central. 
Los neurólogos tienen el reto de evaluar pacientes con enfermedades neurológicas preexistentes que contraen el virus, pacientes con COVID-19 que presentan emergencias neurológicas y pacientes portadores de COVID-19 que desarrollan complicaciones neurológicas secundarias, durante el curso de la enfermedad o posterior a la misma, algunos autores y reportes recientes sugieren que la presencia de manifestaciones neurológicas en pacientes portadores de SARS-CoV-2 puede asociarse a mayor gravedad de la enfermedad.

Respuesta inmunitaria contra SARs-CoV-2 Los coronavirus son una familia de virus ARN que se encuentran en todo el mundo y ocasionan cuadros respiratorios, con frecuencia leves; sin embargo, durante 2002 y 2003, el síndrome respiratorio agudo severo (SARS) afectó a más de 8000 personas en el mundo, con una mortalidad de $10 \%$ y en 2012 el MERS afectó a cerca de 2500 personas, con una mortalidad de $35 \%{ }^{5}$

Los coronavirus reciben su nombre por las proyecciones en forma de corona que presentan al microscopio. En su estructura tienen cuatro proteínas importantes: spike (S), de membrana (M), envelope (E) y nucleocápside $(\mathrm{N})$.

La infección por coronavirus se adquiere por exposición a microgotas que exhalan los individuos infectados o por contacto directo con partículas virales. Esta infección induce autofagia, desprendimiento de la membrana basal e inhibición de la expresión de la proteína de la enzima convertidora de angiotensina 2 (ECA2), que induce daño pulmonar agudo. La liberación de viriones favorece la infección de células vecinas y genera daño sistémico, dado que la proteína ECA2 está distribuida ampliamente en múltiples sitios del organismo como mucosa oral, nasal, pulmonares, huesos, bazo, piel, corazón, arterias, riñones, tejido adiposo, tejido reproductor y sistema nervioso central (SNC [tálamo, cerebelo, núcleo olivar inferior] $)^{5,6,7}$

La infección por SARS-CoV-2 genera una respuesta inmunitaria localizada inicialmente al tracto respiratorio, con reclutamiento de macrófagos, monocitos, liberación de citocinas y formación de una respuesta adaptativa por parte de las células $\mathrm{T}$ y $\mathrm{B}$; en la mayoría de los casos el proceso remite, sin embargo, algunos pacientes evolucionan a formas graves y sistémicas (figura 1 ). ${ }^{8}$

\section{La respuesta inmunológica innata}

La respuesta inmunológica innata está mediada por una interacción compleja entre las células de la respuesta inmune innata (macrófagos, neutrófilos, células dendríticas $[\mathrm{CD}]$ ) y las células epiteliales del órgano blanco.

Cuando el virus ingresa a la célula, su ARN es reconocido por los patrones receptores de reconocimiento de patógenos que se expresan en las células epiteliales y macrófagos alveolares. ${ }^{9}$

Estos receptores de reconocimiento de patógenos incluyen a TLR3, TLR7 y TLR8, activan una cascada de señalización que desencadena el reclutamiento de factores de transcripción como NFkB, IRF3, IRF7 entre otros y amplifica la respuesta inmune innata, a través de las vías JAK-STAT. ${ }^{9}$

Los patrones receptores de reconocimiento de patógenos activados codifican y regulan la transcripción de los interferones (INF) tipos I y III y diversas quimiocinas, cuya función es amplificar la respuesta inmune, atraer más leucocitos de la respuesta innata (neutrófilos, monocitos, células natural killer y $\mathrm{CD}$ ), que a su vez producen más quimiocinas y reclutamiento linfocitario (cuadro 1).

Las CD y macrófagos captan, procesan y presentan fracciones del virus, a través de sus moléculas de histocompatibilidad para que estas sean reconocidas por los linfocitos T. ${ }^{10,11,12,13}$

El SARS-CoV-2, a diferencia de otros coronavirus, tiene la capacidad de eludir los efectos de INF-I e INF-III, que ocasionan pérdida de control viral en la fase temprana de la infección, aparición de edema pulmonar, hipoxia y progresión a fibrosis pulmonar en las fases tardías.

\section{Respuesta inmune adaptativa}

La respuesta inmune adaptativa a SARS-CoV-19 ocurre en los primeros siete a 10 días de la infección.

La memoria de células B y de plasmablastos de expansión se detectan tempranamente en la infección, con secreción de anticuerpos $\operatorname{IgM}$ e $\operatorname{Ig} \mathrm{A}$ entre los días cinco y siete y de IgG entre el día siete y el 10 después del inicio de los síntomas. En general, los títulos séricos de IgM e IgA declinan después de 28 días y los títulos séricos de IgG aproximadamente a los 49 días. $^{13}$

La respuesta humoral induce la formación de anticuerpos, especialmente del isotipo IgG, dirigidos contra el dominio RBD (receptor binding do- 


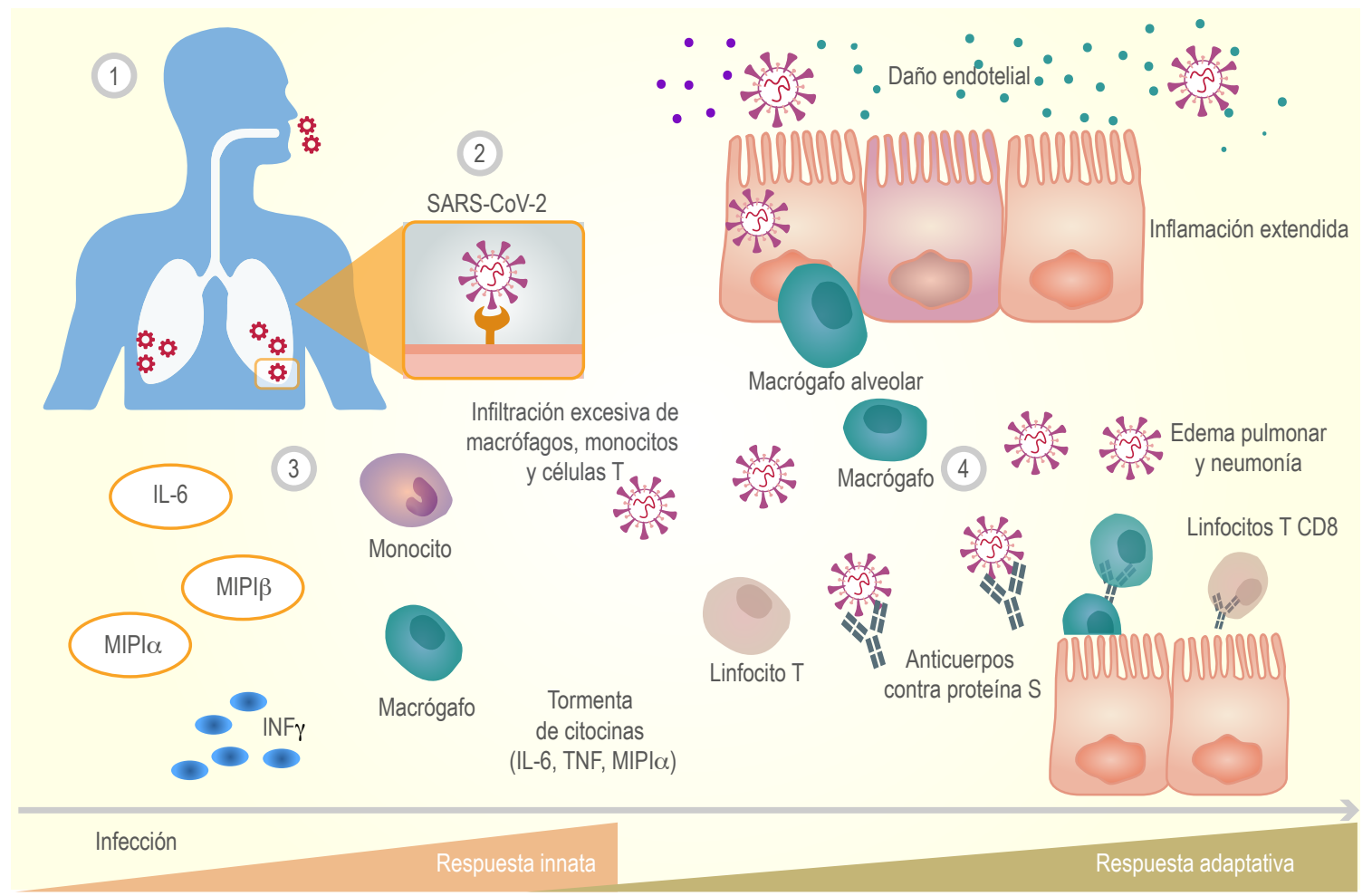

Figura 1. Respuesta inmunitaria durante la infección por SARS-CoV-2. 1. El virus llega a los alveolos pulmonares y al epitelio respiratorio. 2. La proteína $S$ del virus se une al receptor celular ECA2. 3. Macrófagos alveolares y células epiteliales secretan citocina y quimiocinas como IL-6, MIP1 $\alpha$, MIP1 $\beta$ e INF- $\gamma$. 4 . Se ha propuesto que la linfopenia marcada afecta la función de las celulas $T$ y hay una producción disminuida de interferones. Las células T activas contribuyen a la exacerbación de las respuestas inflamatorias, que pueden causar daño pulmonar. Los anticuerpos participan en citotoxicidad mediada por anticuerpos junto con las células T CD8; también los anticuerpos podrían tener una función neutralizante. Estos últimos datos todavía no están completamente claros.

mine) de la glucoproteína $\mathrm{S}$, que inhibe la unión del virus a las proteínas ECA2.

Simultáneamente, SARs-CoV-2 activa a las células $\mathrm{T}$ en la primera semana de infección y la memoria específica de las células CD4+ y CD8+ al virus; alcanza su pico en las primeras dos semanas y permanece en niveles bajos por 100 días. ${ }^{14} \mathrm{La}$ relación entre la carga viral y la respuesta inmunitaria podría influir en la calidad de la respuesta de la célula $\mathrm{T}$; sin embargo, se requieren más estudios para comprender esta relación.

La linfopenia es un indicador de gravedad y suele ser transitoria; afecta a las células T CD4+, T CD8+, células B y natural killer; además, IL-6, IL-10 o TNF $\alpha$ muestran altas concentraciones. Se observa activación de células $\mathrm{T}$ y concentraciones elevadas de moléculas proapoptóticas como FAS (CD95), véase cuadro 2..$^{15,16,17,18}$

\section{Neurotropismo en la infección por \\ SARS-CoV-2}

El neurotropismo es la capacidad de un patógeno para invadir y sobrevivir en el sistema nervioso. Se ha demostrado la afinidad de SARS-CoV-2 por el sistema nervioso; los mecanismos por los cuales puede ocurrir se describen a continuación.

\section{Daño por infección directa}

Se ha detectado material genético y proteínas de varios virus en muestras de tejido del sistema nervioso, que sugiere la probabilidad de que virus pueden invadir directamente el sistema nervioso y causar daño. 


\begin{tabular}{|c|c|c|}
\hline Leucocitos & Cambios durante la infección & Cambio de fenotipo y función \\
\hline Neutrófilos & Se incrementan en la infección severa & $\begin{array}{l}\text { Baja regulación en la desgranulación y las vías } \\
\text { de activación }\end{array}$ \\
\hline Eosinófilos & $\begin{array}{l}\text { Disminuyen en el inicio de la infección } \\
\text { grave y recuperan sus niveles cuando el } \\
\text { paciente se restablece }\end{array}$ & \\
\hline Monocitos & $\begin{array}{l}\text { Se incrementa su migración a pulmón. } \\
\text { Aumentan en sangre periférica durante la } \\
\text { recuperación }\end{array}$ & $\begin{array}{l}\text { Se han identificado pacientes COVID-19 una } \\
\text { población de monocitos CD206+ CD16+ CD11b+ } \\
\text { CD68+ CD163+. Hay un incremento de marca- } \\
\text { dores inflamatorios en los monocitos durante la } \\
\text { recuperación }\end{array}$ \\
\hline Macrófagos & Incremento en pulmón & $\begin{array}{l}\text { Se ha identificado una subpoblación de macrófa- } \\
\text { gos inflamatorios en los pulmones de pacientes } \\
\text { con COVID-19 }\end{array}$ \\
\hline Células natural killer & Disminuyen durante la infección & $\begin{array}{l}\text { Se incrementa la expresión del receptor inhibidor } \\
\text { NKG2A. Se observa trastornos producción de } \\
\text { citocinas en pacientes con infección severa }\end{array}$ \\
\hline
\end{tabular}

Propagación por la circulación sanguínea o la barrera hematoencefálica

La barrera hematoencefálica se compone del endotelio vascular, astrocitos, pericitos y matriz extracelular. El endotelio de nuestro organismo expresa ECA2 y por esta razón tiene riesgo de ser invadido por SARS-CoV-2. Se ha demostrado la presencia de partículas virales de SARS-CoV-2 en el endotelio capilar y neuronas del lóbulo frontal, las cuales contienen partículas virales; la microscopia electrónica muestra, además, endocitosis y exocitosis de partículas virales a través de las células endoteliales. ${ }^{19,20}$

Una vez que el virus tiene acceso al tejido vascular y neuronal inicia un nuevo ciclo de replicación, daño del tejido vascular y neuronal, al entrar en contacto con la ECA2 de las neuronas, la glía y los vasos.

Una liberación secundaria a la circulación sanguínea puede incrementar la permeabilidad de la barrera hematoencefálica a través de la producción de citocinas y promover la entrada del virus al cerebro y causar encefalitis viral.

El proceso de inflamación sistémica que caracteriza a COVID-19 aumenta la permeabilidad de la barrera hematoencefálica y permite que las células inmunes infectadas, citocinas y posiblemente el virus se introduzcan al SNC. ${ }^{21}$
Diseminación trans-sináptica y daño neuronal La vía neuronal es el mecanismo más importante para que los virus neurotrópicos ingresen al $\mathrm{SNC}$ a través del transporte neuronal anterógrado o retrógrado a través de proteínas motoras como dineínas y quinesinas. ${ }^{10}$

El ingreso directo a través del nervio olfatorio y la lámina cribosa es otro mecanismo potencial del SARs-CoV-2 para entrar al SNC (figura 2).

La organización anatómica única del bulbo y nervios olfatorios en la cavidad nasal y frontal anterior es un canal expedito y directo entre el epitelio nasal y el SNC. El SARS-COV-2 puede ingresar al cerebro vía el tracto olfatorio y posteriormente alcanzar el parénquima cerebral y el líquido cefalorraquídeo a través del nervio y bulbo olfatorios en los siguientes siete días y generar reacción de inflamación y desmielinización. ${ }^{22,23}$

En Italia, Politi et al. informaron sobre una técnica radióloga de 25 años que laboraba en un área COVID, quien presentó tos seca leve y posteriormente anosmia y disgeusia persistente y severa sin datos respiratorios. La resonancia magnética de cráneo mostró hiperintensidad cortical en el giro recto derecho e hiperintensidad sutil en el bulbo olfatorio, con prueba de PCR positiva para SARS-CoV-2; 28 días después tuvo remisión de la anosmia, disgeusia y de las alteraciones de la re- 


\begin{tabular}{|c|c|c|}
\hline Linfocitos & Cambio en la frecuencia durante la infección & Cambio de fenotipo y función \\
\hline Células T CD4 & $\begin{array}{l}\text { Disminuyen en pacientes con enfermedad grave. Hay } \\
\text { aumento de las células T CCR6+ CD4+ en enfermedad } \\
\text { grave. Durante la recuperación se recupera su número }\end{array}$ & $\begin{array}{l}\text { Disminuye la producción de INF-y y se } \\
\text { incrementa TNFa }\end{array}$ \\
\hline Células T CD8 & $\begin{array}{l}\text { Disminuyen en la infección grave en sangre periférica. } \\
\text { Hay expansión clonal en los pulmones en pacientes con } \\
\text { enfermedad grave. Su número vuelve a la normalidad } \\
\text { durante la recuperación del paciente }\end{array}$ & $\begin{array}{l}\text { Mayor expresión de CD25, PD-1, gran- } \\
\text { zima B y perforinas. Respuesta de INF } \\
\text { elevada en pacientes con enfermedad } \\
\text { grave }\end{array}$ \\
\hline Células B & Su número y centros germinales se incrementan & $\begin{array}{l}\text { Aumento de plasmablastos, dismi- } \\
\text { nuyen las células B de memoria y } \\
\text { aumentan las células B transicionales }\end{array}$ \\
\hline Células T & Disminuyen & \\
\hline
\end{tabular}

sonancia magnética. Estos hallazgos sugieren que SARS-CoV-2 puede invadir el cerebro a través de la vía olfatoria y causar disfunción olfatoria de origen sensorineural. ${ }^{24}$

\section{Daño hipóxico}

SARS-CoV-2 induce disfunción y falla en el intercambio alveolar de gases y produce en forma secundaria hipoxia en el SNC por aumento en el meta- bolismo anaeróbico en las mitocondrias de células cerebrales, con presencia de vasodilatación, edema, edema intersticial, obstrucción del flujo sanguíneo cerebral y clínicamente puede manifestarse como cefalea secundaria a isquemia.

Si la hipoxia persiste, el trastorno circulatorio y el edema cerebral pueden empeorar y generar hipertensión endocraneana, manifestada como somnolencia, edema bulbar y coma o muerte. ${ }^{12}$

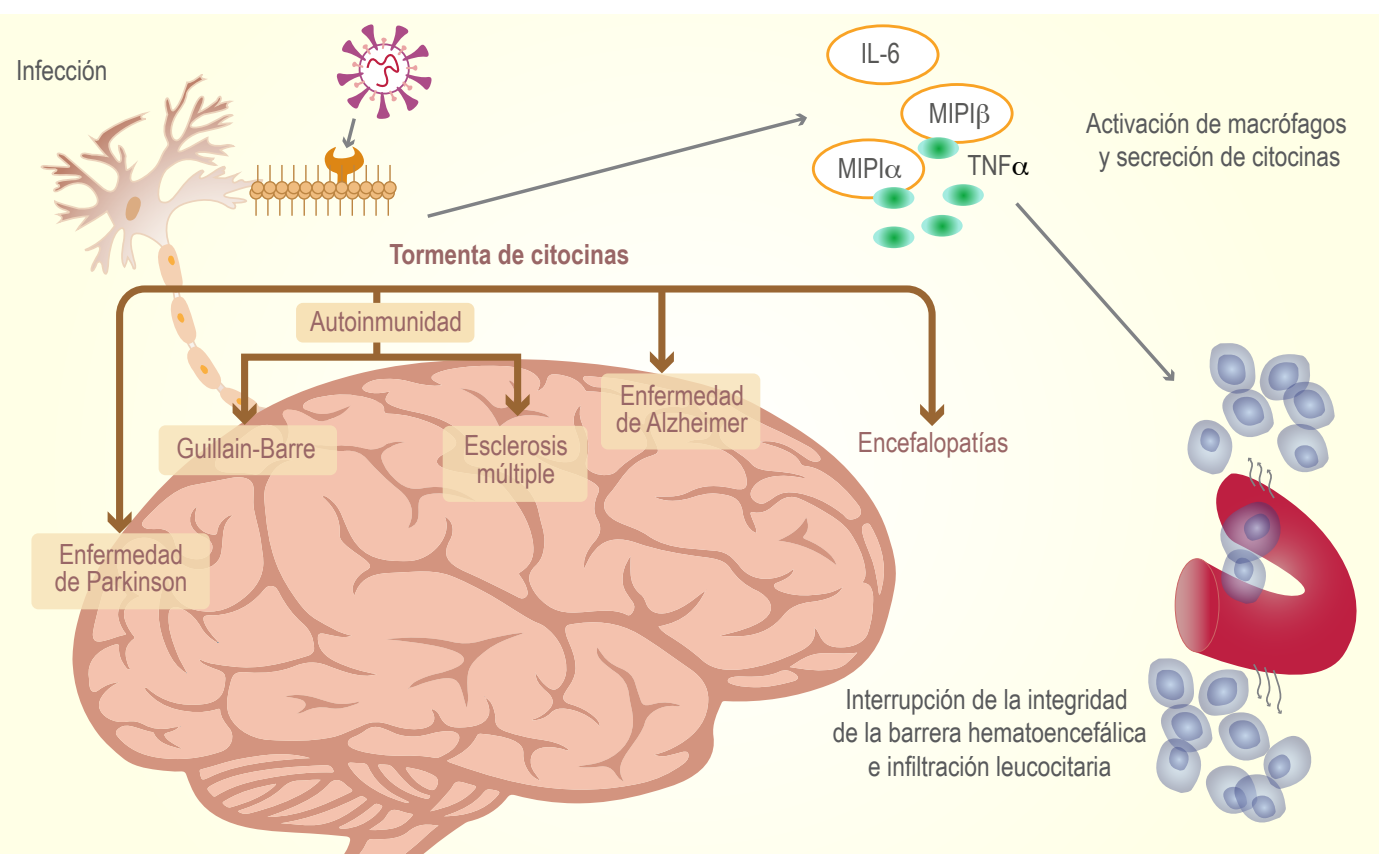

Figura 2. Los mecanismos patológicos de la participación del SNC mediada por el sistema inmunitario en la infección por SARS-CoV-2. Después de la infección de SARS-CoV-2 en el SNC, se activan los macrófagos con liberación extrema de citocina y quimiocinas e infiltración de linfocitos en el SNC, por lo tanto se manifiestan diversos trastornos neurológicos. 
Enzima convertidora de angiotensina 2

y el neurotropismo

Los receptores ECA2 dentro del cerebro humano se expresan en neuronas, astrocitos, oligodendrocitos, sustancia negra, ventrículos, giro temporal medial, corteza posterior del cíngulo y bulbo olfatorio.

Se ha identificado la expresión de ECA2 en corteza motora, citoplasma de neuronas, células gliales y vías simpáticas del tallo cerebral.

SARS-CoV-2 también puede interactuar con la ECA2 expresada en el endotelio capilar, con daño a la barrera hematoencefálica, ingresando al SNC atacando el sistema vascular.

\section{La neurología del COVID-19}

Las manifestaciones neurológicas de la infección por SARs-CoV-2 cada vez se reportan con más frecuencia; los esfuerzos de los neurólogos están dirigidos al oportuno control de los síntomas y de los mecanismos responsables de ello y proporcionar el tratamiento más adecuado.

Desde el reporte inicial del estudio realizado en China con 214 pacientes hospitalizados por COVID-19, los resultados muestran que hasta un tercio de ellos presentaban manifestaciones neurológicas que incluían alteraciones del estado mental y enfermedad cerebral vascular aguda, espacialmente los pacientes con enfermedad respiratoria severa. ${ }^{25}$

Los reportes actuales incluyen, además, otros síntomas y signos como anosmia, ageusia, encefalopatía hemorrágica necrotizante aguda, encefalopatía tóxica-metabólica, cefalea, mialgia, insuficiencia respiratoria central, mielitis, ataxia y diversas manifestaciones neuropsiquiátricas.

Algunos autores, refieren otros datos neurológicos: cefalea, mareos, vértigo, alteración de la conciencia, deterioro neurológico focal, compromiso de nervios craneales, crisis convulsivas y, en menor proporción, meningoencefalitis, encefalopatía necrosante aguda severa, edema cerebral y enfermedad vascular cerebral (EVC) (cuadro 3).

Se ha descrito la frecuencia de alteraciones neurológicas que se manifiestan en el gusto-olfato $(35.6 \%)$, mialgias $(18.5 \%)$, cefalea $(10.7 \%)$, enfermedad vascular $(8.1 \%)$, alteraciones del estado mental ( $7.8 \%$ ), convulsiones $(1.5 \%)$ y otras menos frecuentes: ataxia, neuralgia, síndrome de GuillainBarré, síndrome de Miller Fisher, hemorragia intracerebral, polineuritis. ${ }^{26}$
Cuadro 3. Principales manifestaciones neurológicas en COVID-19

Sistema nervioso periférico

- Hiposmia o anosmia

- Disgeusia

- Debilidad general

- Neuropatía craneal

- Polineuropatía

- Síndrome de Guillian-Barré

Sistema nervioso central

- Cefalea

- Vértigo

- Enfermedad vascular cerebral

- Crisis convulsivas

- Estado epiléptico

- Confusión

- Delirio

- Estupor

- Coma

- Encefalitis

\section{Otras manifestaciones}

- Mialgias

- Encefalomielitis

- Encefalitis autoinmune

La presencia de síntomas neurológicos como EVC, deterioro del estado de conciencia y lesiones músculo esqueléticas se han asociado a mal pronóstico.

\section{Anosmia y augeusia}

La ausencia o disminución de la olfación (hiposmia o anosmia) y del sentido del gusto (hipogeusia, disgeusia) son síntomas comunes entre pacientes con COVID-19 y se ha propuesto también que pueden ser indicativos del potencial efecto neurotrópico del coronavirus para invadir el cerebro. ${ }^{27}$

En el estudio chino referido anteriormente, que incluyó a 214 pacientes con COVID-19, $5.6 \%$ presentaban hipogeusia y $5.1 \%$ hiposmia. ${ }^{25}$

En otro estudio europeo multicéntrico de 417 pacientes, se refiere una frecuencia de disfunción olfatoria de $85 \%$ y de disfunción gustativa de $88 \%$ en los pacientes estudiados. Estas alteraciones ocurrieron de forma muy temprana en $11.8 \%$ de los casos y la recuperación de la olfacción aconteció en $44 \%$ de los casos. ${ }^{23}$ 


\section{Cefalea}

La cefalea es una de las molestias iniciales más comunes en pacientes con COVID-19. Aunque la prevalencia varía en los distintos reportes, se ha propuesto que puede afectar hasta una tercera parte de los pacientes. Su presencia se ha asociado a mecanismos neuroinflamatorios secundarios a la presencia de citocinas y quimiocinas que estimulan las neuronas sensoriales nociceptivas y producen dolor. ${ }^{28,29,30,31,32,33}$

\section{Enfermedad vascular cerebral (EVC) aguda}

Múltiples estudios demuestran que pacientes con COVID-19 pueden desarrollar EVC isquémico agudo, aunque la frecuencia exacta y los factores de riesgo asociados no son claros. Aunque algunos reportes describen casos de EVC con formas leves de COVID-19, la gran mayoría de los casos de EVC se asocia con formas moderadas o severas de COVID-19. En un estudio el riesgo de EVC isquémico en pacientes con infección por SARS-CoV-2 severo $(\mathrm{n}=31)$ fue más alto que en pacientes con influenza estacional $(\mathrm{n}=3,1.6 \%$ versus $0.2 \%$, razón de momios $=7.6$, IC $95 \%=2.3-25.2) ;{ }^{31}$ la mortalidad fue de $38 \%$. En otro reporte, Mao et al. describieron cinco pacientes con EVC ( $80 \%$ isquémicos) que tenían formas graves de COVID-19 con aumento en los niveles de dímero $\mathrm{D}$, trombocitopenia y afectación múltiple de órganos. ${ }^{25}$

Los mecanismos de la EVC en COVID-19 son numerosos. El estado hipercoagulable que acompaña a las formas severas de la enfermedad es el mayor factor observable, así como la coexistencia de complicaciones cardiacas que llevan a la formación de fuentes embólicas.

La infección por SARs-CoV-2 puede causar "tormenta de citocinas", que puede ser uno de los factores asociados al desarrollo de EVC. Estos pacientes cuando cursan con infección severa y en estado crítico presentan niveles elevados de dímero $\mathrm{D}$ y reducción importante de plaquetas, lo que puede predisponer a EVC. . $^{32,33,34,35,36,37,38,39}$

El manejo de pacientes con COVID-19 y EVC constituye un reto que requiere mayores investigaciones. Los protocolos hospitalarios para reconocer rápidamente $\mathrm{EVC}$ se han complicado por la coexistencia de eventos respiratorios y otras comorbilidades en los pacientes con COVID-19, los cuales pueden requerir sedación profunda, sobre todo los casos severos, lo que dificulta el reconocimiento de cambios neurológicos. Las redes diseñadas para la evaluación y triaje de EVC y el envío hacia centros donde pueda efectuarse el tratamiento inmediato del EVC agudo (incluyendo trombectomía) no están funcionando debido a la fractura de los protocolos de transferencia en sistemas hospitalarios que han estado al límite de su capacidad por la pandemia.

Las estrategias de prevención secundaria, incluida la administración de medicamentos antitrombóticos, también deberán ser modificados en los pacientes que en forma incrementada presentan complicaciones isquémicas y hemorrágicas.

Por otro lado, llama la atención que a pesar del reconocimiento cada día mayor de EVC como una complicación de COVID-19, la hospitalización por este motivo haya disminuido sustancialmente durante la pandemia, como se ha demostrado en reportes de múltiples regiones y en un análisis de un estudio radiológico nacional de software de tomografía computada perfusoria. La disminución de pacientes que presentan EVC agudo, la cual no tiene una explicación clara, se acompaña de un decremento en las evaluaciones de urgencia y representa probablemente el temor de los pacientes a contagiarse en hospitales y servicios de urgencia.

El efecto de dicho retraso en la atención y manejo del EVC en pacientes es difícil de cuantificar, pero dada la mejoría sustancial ya conocida en el manejo del EVC agudo, es probable que en el futuro mediato veamos los efectos tardíos en el EVC como una secuela de esta pandemia. ${ }^{40}$

\section{Encefalitis viral}

SARS-CoV-2 puede invadir el SNC e inducir la presencia de enfermedades como la encefalitis. Existe escasa evidencia de la presencia de encefalitis secundaria a SARS-CoV-2. El diagnóstico temprano de encefalitis viral es crítico.

En Wuhan se confirmó la presencia de SARSCoV-2 en el líquido cefalorraquídeo de un paciente con COVID-19 al secuenciar el genoma, lo que confirmó la sospecha clínica de encefalitis viral por COVID-19; el paciente presentó cefalea, fiebre y crisis. La resonancia magnética reveló restricción en la difusión del lóbulo temporal derecho, atrofia hipocampal y ventriculitis; además, se detectó ARN de SARS-CoV-2 en el líquido cefalorraquídeo. No queda claro si alguna de las manifestaciones reportadas en el paciente se debió a causas distintas a encefalitis. ${ }^{41}$ 
Encefalopatia necrosante hemorrágica aguda Recientemente, Poyiadji et al. reportaron un caso de encefalopatía aguda en una mujer mayor de 50 años con encefalopatía aguda e infección por SARSCoV-2, corroborada por prueba de PCR positiva tomada de nasofaringe. El estudio del líquido cefalorraquídeo fue negativo para bacterias, herpes simple 1 y 2 , virus de varicela zoster y virus del Nilo del oeste. La resonancia magnética con gadolinio como medio de constrate demostró lesiones hemorrágicas redondeadas con reforzamiento en anillo en el tálamo, lóbulos temporales mediales y regiones subinsulares. Los autores indicaron que estos hallazgos sugieren la presencia de encefalopatía necrosante hemorrágica aguda ${ }^{42}$

Se ha indicado que la encefalopatía necrosante hemorrágica aguda causa "tormenta de citocinas", generando elevación de citocinas proinflamatorias como interleucina (IL) 6, factor de necrosis tumoral-alfa (TNF $\alpha$ ), IL-10, IL-15, IL-1B, TNF soluble al receptor e interferón gamma más linfocitos, en particular CD56+, células natural killer que conllevan daño cerebral mediante alteraciones de la barrera hematoencefálica por tripsina y metloproteasa-9 de la matriz sin ruptura de la pared del vaso. ${ }^{43,44,45}$

\section{Otras}

Las complicaciones neurológicas del COVID-19 no se limitan al SNC. Al igual que con otros virus
SARS, han emergido reportes de complicaciones neuromusculares por SARS-CoV-2, incluyendo casos de síndrome de Guillain-Barré y problemas musculares manifestados por mialgias y niveles séricos de CPK. Es probable que sea poco reportado el compromiso del sistema nervioso periférico; además, se requieren estudios de neurofisiología para determinar la incidencia y las características fidedignas de estas manifestaciones. En el caso de la polineuropatía en el contexto de COVID-19, esta tiene un curso temprano y sigue un patrón parainfeccioso. La naturaleza neuroinvasiva del virus SARS-CoV-2, favorecida por un entorno inflamatorio, desencadena una respuesta inmunológica aberrante, secundaria al mimetismo molecular que conduce a la desmielinización periférica. ${ }^{38,46,47,48}$

\section{Conclusión}

Es importante dilucidar los defectos de la respuesta inmune que ocurren en los individuos infectados por SARs-CoV-2 y que dan como resultado una baja respuesta protectora o liberación excesiva de citocinas que conllevan a inflamación severa y falla multisistémica. Una mejor comprensión de estos eventos contribuiría al diseño de abordajes terapéuticos y delimitación de biomarcadores pronósticos que posiblemente eviten secuelas, como las neurológicas, que impidan la restauración a una vida personal, laboral o escolar normal y generen impacto económico a las instituciones de salud.

\section{Referencias}

1. Organización Mundial de Is Salud [Internet]. Ginebra, Suiza: Brote de enfermedad por coronavirus (COVID-19). Disponible en: https://www.who.int/es/emergencies/diseases/novel-coronavirus

2. COVID-19 Dashboard by the Center for Systems Science and Engineering (CSSE) at Johns Hopkins University \& Medicine [Internet]. EE. UU.: Johns Hopkins University \& Medicine; c2021. Disponible en: https://coronavirus.jhu.edu/map.html

3. Guan W, Ni Z, Hu Y, Liang W, Ou C, He J, et al. Clinical characteristics of coronavirus disease 2019 in China. N Engl J Med. 2020;382(18):1708-1720. DOI: 10.1056/NEJMoa2002032

4. Majid F, Ali M, Somayeh M, Cyrus AR. Neurobiology of COVID-19. J Alzheimers Dis. 2020;76(1):3-19. DOI: 10.3233/JAD-200581

5. Machhi J, Herskovitz J, Senan AM, et al. The natural history, pathobiology, and clinical manifestations of SARS-CoV-2 infections. J Neuroimmune Pharmacol. 2020;1-28. DOI: 10.1007/s11481-020-09944-5

6. Mohammadi S, Moosaie F, Aarabi MH. Understanding the immunologic characteristics of neurologic manifestations of SARS-CoV-2 and potential immunological mechanisms. Mol Neurobiol. 2020;57(12):5263-5275. DOI: 10.1007/s12035-020-02094-y 
7. Shi Y, Wang Y, Shao C, Huang J, Gan J, Huang X, et al. COVID-19 infection: the perspectives on immune responses. Cell Death Differ. 2020;27(5):1451-1454. DOI: 10.1038/s41418-020-0530-3

8. Han R, Huang L, Jiang H, Dong J, Peng H, Zhang D. Early clinical and CT manifestations of coronavirus disease 2019 (COVID-19) pneumonia. AJR Ame J Roentgenol. 2020;215(2):338-343. DOI: 10.2214/ AJR.20.22961

9. García LF. Immune response, inflammation, and the clinical spectrum of COVID-19. Front Immunol. 2020;11.1441. DOI: 10.3389/fimmu.2020.01441

10. Chong K, Mehta PR, Shukla G, Mehta AR. COVID-19, SARS and MERS: a neurological perspective. J Clin Neurosci. 2020;77:13-16. DOI: 10.1016/j.jocn.2020.04.124

11. Cheng Q, Yang Y, Gao J. Infectivity of human coronavirus in the brain. EBioMedicine. 2020;56:102799. DOI: 10.1016/j.ebiom.2020.102799

12. Li H, Xue $Q, X u X$. Involvement of the nervous system in SARS-CoV-2 infection. Neurotox Res. 2020;38(1):1-7. DOI: 10.1007/s12640-020-00219-8

13. Kursat-Azkur A, Akdis M, Sokolowska M, van de Veen W, Brüggen C, O'Mahony L, et al. Immune response to SARS-CoV-2 and mechanisms of immunopathological changes in COVID-19. Allergy. 2020;75(7):1564-1581. DOI: 10.1111/all.14364

14. Guihot A, Litvinova E, Autran B, Debré P, Vieillard V. Cell-mediated immune responses to COVID-19 infection. Front Immunol. 2020;11:1662. DOI: 10.3389/fimmu.2020.01662

15. Kim SY, Kim DW. Does the clinical spectrum of coronavirus disease 2019 (COVID-19) show regional differences? Clin Exp Otorhinnolaryngol. 2020;13(2):83-84. DOI: 10.21053/ceo.2020.00612

16. Abboud H, Abboud FZ, Kharbouch H, Arkha Y, Abbadi NE, Ouahabi AE. COVID-19 and SARS-CoV-2 infection: pathophysiology and clinical effects on the nervous system. World Neurosurg. 2020;140:49-53. DOI: 10.1016/j.wneu.2020.05.193

17. Zhou Z, Kang H, Li S, Zhao X. Understanding the neurotropic characteristics of SARS-CoV-2: from neurological manifestations of COVID-19 to potential neurotropic mechanisms. J Neurol. 2020;267(8):21792184. DOI: 10.1007/s00415-020-09929-7

18. Fathi N, Rezaei N. Lymphopenia in COVID-19: therapeutic opportunities. Cell Biol Int. 2020;44(9):17921797. DOI: 10.1002/cbin.11403

19. Koralnik IJ, Kenneth LT. COVID-19: a global threat to the nervous system. Ann Neurol. 2020;88(1):1-11. DOI: 10.1002/ana.25807

20. Asadi-Pooya AA, Simani L. Central nervous system manifestations of COVID-19: a systematic review. J Neurol Sci. 2020;413:116832. DOI: 10.1016/j.jns.2020.116832

21. Brouwer MC, Ascione T, Pagliano P. Neurologic aspects of COVID-19: a concise review. Infez Med. 2020;28(Suppl 1):42-45. Disponible en: https://www.infezmed.it/media/journal/Vol_28_suppl1_2020_7.pdf

22. Ellul MA, Benjamin L, Singh B, Lant S, Michael BD, Easton A, et al. Neurological associations of COVID-19. Lancet Neurol. 2020;19(9):767-783. DOI: 10.1016/S1474-4422(20)30221-0

23. Lechien JR, Chiesa-Estomba CM, de Siati DR, Horoi M, D Le Bon S, Rodriguez A, et al. Olfactory and gustatory dysfunctions as a clinical presentation of mild-to-moderate forms of the coronavirus disease (COVID-19): a multicenter European study. Eur Arch Otorhinolaryngol. 2020;277(8):2251-2261. DOI: 10.1007/s00405-020-05965-1

24. Politi LS, Salsano E, Grimaldi M. Magnetic resonance imaging alteration of the brain in a patient with coronavirus disease 2019 (COVID-19) and anosmia. JAMA Neurol. 2020;77(8):1028-1029. DOI: 10.1001/ jamaneurol.2020.2125

25. Mao L, Jin H, Wang M, Hu Y, Chen S, Quanwei H, et al. Neurologic manifestations of hospitalized patients with coronavirus disease 2019 in Wuhan, China. JAMA Neurol. 2020;77(6):683-690. DOI: 10.1001/ jamaneurol.2020.1127

26. Tsai ST, Lu MK, San S, Tsai CH. The neurologic manifestations of coronavirus disease 2019 pandemic: a systemic review. Front Neurol. 2020;11:498. DOI: 10.3389/fneur.2020.00498 
27. Xiangming Meng, Yanzhong Deng, Zhiyong Dai, Zhisheng Meng. COVID-19 and anosmia: a review based on up-to-date knowledge. Am J Otolaryngol. 2020;41(5):102581. DOI: 10.1016/j.amjoto.2020.102581

28. Bolay H, Gül A, Baykan B. COVID-19 is a real headache! Headache. 2020:10.1111/head.13856. DOI: 10.1111/head.13856

29. Yavarpour-Bali H, Ghasemi-Kasman M. Update on neurological manifestations of COVID-19. Life Sci. 2020;257:118063. DOI: 10.1016/j.lfs.2020.118063

30. Correia AO, Feitosa PWG, Moreira JLDS, Nogueira SÁR, Fonseca RB, Nobre MEP. Neurological manifestations of COVID-19 and other coronaviruses: A systematic review. Neurol Psychiatry Brain Res. 2020;37:27-32. DOI: 10.1016/j.npbr.2020.05.008

31. Merkler A, Parikh N, Mir S, Gupta A. Risk of ischemic stroke in patients with coronavirus disease 20019 (COVID19) vs patients with Influenza. JAMA Neurol. 2020;77(11):1-7. DOI: 10.1001/ jamaneurol.2020.2730

32. Ferrarese C, Silani V, PrioriA, Galimberti S, Agostoni E, Monaco S, et al. An Italian multicenter retrospectiveprospective observational study on neurological manifestations of COVID-19 (NEUROCOVID). Neurol Sci. 2020;41(6):1355-1359. DOI:10.1007/s10072-020-04450-1

33. Vacchiano V, Riguzzi P, Volpi L, Tappatà M, Avoni P, Rizzo G, et al. Early neurological manifestations of hospitalized COVID-19 patients. Neurol Sci. 2020 Aug;41(8):2029-2031. DOI:10.1007/s10072-02004525-z

34. Sharifian-Dorche M, Huot P, Osherov M, Wen D, Saveriano A, Giacomini PS, et al. Neurological complications of coronavirus infection; a comparative review and lessons learned during the COVID-19 pandemic. J Neurol Sci. 2020;417:117085. DOI: 10.1016/j.jns.2020.117085

35. Nepal G, Rehrig JH, Shrestha GS, Shing YK, Yadav JK, Ojha R, et al. Neurological manifestations of COVID-19: a systematic review. Crit Care. 2020;24(1):421. DOI: 10.1186/s13054-020-03121-z

36. Pinna P, Grewal P, Julianne P, Hall JP, Tavarez T, Dafer RM, et al. Neurological manifestations and COVID-19: experiences from a tertiary care center at the Frontline. J Neurol Sci. 2020;415:116969. DOI: 10.1016/j.jns.2020.116969

37. Wang L, Shen Y, Li M, Chuang H, Ye Y, Zhao H, et al. Clinical manifestations and evidence of neurological involvement in 2019 novel coronavirus SARS-CoV-2: a systematic review and meta-analysis. J Neurol. 2020;267(10):2777-2789. DOI: 10.1007/s00415-020-09974-2

38. Lechien JR, Chiesa-Estomba CM, Place S, van Laethem Y, Cabaraux P, Mat Q, et al. Clinical and epidemiological characteristics of 1420 European patients with mild-to-moderate coronavirus disease 2019. J Intern Med. 2020;288(3):335-344. DOI: 10.1111/joim.13089

39. Romero-Sánchez CM, Díaz-Maroto I, Fernández-Díaz E, Sánchez-Larsen Á, Layos-Romero A, GarcíaGarcía J, et al. Neurologic manifestations in hospitalized patients with COVID-19: the ALBACOVID registry. Neurology. 2020;95(8):e1060-e1070. DOI: 10.1212/WNL.0000000000009937

40. Divani AA, Andalib S, di Napoli M, Lattanzi S, Shazam-Hussain M, Biller J, et al. Coronavirus disease 2019 and stroke: clinical manifestations and pathophysiological insights. J Stroke Cerebr Dis. 2020;29(8):104941. DOI: 10.1016/j.jstrokecerebrovasdis.2020.104941

41. Moriguchi T, Harii N, Goto J, Harada D, Sugawara H, Takamino J, et al. A first case of meningitis/encephalitis associated with SARS-coronavirus-2. Int J Infect Dis. 2020;94:55-58. DOI: 10.1016/j.jij.2020.03.062

42. Poyiadji N, Shahin G, Noujaim D, Stone M, Patel S, Griffith B, et al. COVID-19-associated acute hemorrhagic necrotizing encephalopathy: imaging features. 2020;296(2):E119-E120. DOI: 10.1148/ radiol.2020201187

43. Krett J, Jewett GA, Elton-Lacasse C, Fonseca K, Hahn C, Selena A, et al. Hemorrhagic encephalopathy associated with COVID-19. J Neuroimmunol. 2020;346:577326. DOI: 10.1016/j. jneuroim.2020.577326

44. Dixon L, Varley J, Gontsarova A, Mallon D, Tona F, Muir D, et al. COVID-19-related acute necrotizing encephalopathy with brain stem involvement in a patient with aplastic anemia. Neurol Neuroimmunol Neuroinflamm. 2020;7(5):e789. DOI: 10.1212/NXI.0000000000000789 
45. Safaa Saeed Al Mazrouei, Ghufran Aref Saeed, Abeer Ahmed Al Helali, Manzoor Ahmed. COVID-19associated encephalopathy: neurological manifestation of COVID-19. Radiol Case Rep. 2020;15(9):16461649. DOI: 10.1016/j.radcr.2020.07.009

46. Orsucci D, Caldarazzo-lenco E, Nocita G, Napolitano A, Vista M. Neurological features of COVID-19 and their treatment: a review. Drugs Context. 2020;9:2020-5-1. DOI: 10.7573/dic.2020-5-1

47. Kilinc D, van de Pasch S, Doets AY, Jacobs BC, van Vliet J, Garssen MPJ. Guillain-Barré syndrome after SARS-CoV-2 infection. Eur J Neurol. 2020;27(9):1757-1758. DOI: 10.1111/ene.14398.

48. Dalakas MC. Guillain-Barré syndrome: the first documented COVID-19-triggered autoimmune neurologic disease More to come with myositis in the offing. Neurol Neuroimmunol Neuroinflamm. 2020;7(5):e781. DOI: 10.1212/NXI.0000000000000781 